\title{
Novel G1481V and Q1491H SCN5A mutations linked to long QT syndrome destabilize the Nav1.5 inactivation state
}

\author{
Quentin Plumereau ${ }^{1}$, Olivier Theriault ${ }^{1}$, Valerie Pouliot ${ }^{1}$, Adrien Moreau ${ }^{1}$, Elodie Morel ${ }^{2}$, \\ Veronique Fressart ${ }^{3}$, Isabelle Denjoy ${ }^{4}$, Antoine Delinière ${ }^{5}$, Francis BESSIERE ${ }^{6}$, Philippe \\ Chevalier $^{7}$, Tamer Gamal El-Din ${ }^{8}$, and Mohamed Chahine ${ }^{1}$ \\ ${ }^{1}$ Laval University Faculty of Medicine \\ ${ }^{2}$ Hopices Civils de Lyon \\ ${ }^{3}$ Centre de génétique moléculaire et chromosomique, \\ ${ }^{4}$ Hôpital Bichat - Claude-Bernard Service de Cardiologie \\ ${ }^{5}$ Hopital Cardio-vasculaire et Pneumologique Louis Pradel \\ ${ }^{6}$ Hopital Louis Pradel \\ ${ }^{7}$ Hopital Cardiologique \\ ${ }^{8}$ University of Washington
}

September 11, 2020

\begin{abstract}
BACKGROUND: Nav1.5, which is encoded by the SCN5A gene, is the predominant voltage-gated Na+ channel in the heart. Several mutations of this gene have been identified and have been reported to be involved in several cardiac rhythm disorders, including type 3 long QT syndrome (LQT3), that can cause sudden cardiac death. We analyzed the biophysical properties of two novel variants of the Nav1.5 channel (Q1491H and G1481V) detected in 5- and 12-week-old infants diagnosed with a prolonged QT interval. METHODS: The Nav1.5 wild-type (WT) and the Q1491H and G1481V mutant channels were reproduced in vivo. WT or the mutant channels were co-transfected in HEK 293 cells with the beta 1 regulatory subunit. $\mathrm{Na}+$ currents were recorded using the whole-cell configuration of the patch-clamp technique. RESULTS: The Q1491H mutant channel exhibited a lower current density, a persistent Na+ current, an enhanced window current due to a +20 - $\mathrm{mV}$ shift of steady-state inactivation, a $+10-\mathrm{mV}$ shift of steady-state activation, a faster onset of slow inactivation, and a recovery from fast inactivation with fast and a slow time constants of recovery. The G1481V mutant channel exhibited an increase in current density and $\mathrm{a}+7-\mathrm{mV}$ shift of steady-state inactivation. The observed defects are characteristic of gain-of-function mutations typical of LQT3. DISCUSSION AND CONCLUSION: The 5- and 12-week-old infants displayed prolonged QT intervals. Our analyses of the Q1491H and G1481V mutations correlated with the clinical diagnosis. The observed biophysical dysfunctions associated with both mutations were most likely responsible for the sudden deaths of the two infants.
\end{abstract}

Novel G1481V and Q1491H SCN5A mutations linked to long QT syndrome destabilize the $\mathrm{Na}_{\mathrm{v}} 1.5$ inactivation state

Plumereau Q. ${ }^{1}$, Theriault O. ${ }^{1}$, Pouliot V. ${ }^{1}$, Moreau A. ${ }^{2}$, Morel E. ${ }^{3}$, Fressart V. ${ }^{4}$, Denjoy I. ${ }^{5}$, Delinière $\mathrm{A}^{3}{ }^{3}$, Bessière $\mathrm{F}^{3}$, Chevalier P. ${ }^{3,6,7}$, Gamal El-Din TM. ${ }^{8}$ and ${ }^{*}$ Chahine M. ${ }^{1,9}$

${ }^{1}$ CERVO Brain Research Center, Quebec City, QC, Canada

${ }^{2}$ Inserm U1046, CNRS UMR 9214, Université de Montpellier, Montpellier, France

${ }^{3}$ Lyon Reference Center for Inherited Arrhythmias, Louis Pradel Cardiovascular Hospital, Bron, France 
${ }^{4}$ Centre de génétique moléculaire et chromosomique, Hôpital Pitié-Salpêtrière, Paris, France

${ }^{5}$ Hôpital Bichat Claude Bernard, Paris, France

${ }^{6}$ Department of Rhythmology, Louis Pradel Cardiovascular Hospital, Lyon, France

${ }^{7}$ Université de Lyon, Lyon, France

${ }^{8}$ Department of Pharmacology, University of Washington, Seattle, WA 98195, USA.

${ }^{9}$ Department of Medicine, Faculty of Medicine, Université Laval, Quebec City, QC, Canada

\section{Corresponding author:}

*Mohamed Chahine

CERVO Brain Research Centre

2601, de la Canardiere

Quebec, Qc, Canada

G1J 2G3

418-663-5741 (phone)

418-663-5971 (FAX)

Mohamed.Chahine@phc.ulaval.ca

Short title: G1481V and Q1491H LQT3 mutations disrupt Nav1.5 inactivation

\section{Abbreviations:}

AP Action potential

BS Brugada syndrome

cDNA Complementary DNA

DI-DIV Sodium channel domain 1-4

ECG Electrocardiogram

EADs early after depolarizations

HEK293 Human embryonic kidney cell line

LQTS Long QT interval syndrome

LQT3 Long QT interval syndrome type 3

$P C C D$ progressive cardiac conduction disorder

QTc QT interval corrected for heart rate

SIDS sudden infant death syndrome.

TTX Tetrodotoxin

VT / VF Ventricular tachycardia / ventricular fibrillation

WT Wild-type

Background: $\mathrm{Na}_{\mathrm{v}} 1.5$, which is encoded by the $S C N 5 \mathrm{~A}$ gene, is the predominant voltage-gated $\mathrm{Na}^{+}$channel in the heart. Several mutations of this gene have been identified and have been reported to be involved in several cardiac rhythm disorders, including type 3 long QT syndrome (LQT3), that can cause sudden 
cardiac death. We analyzed the biophysical properties of two novel variants of the $\mathrm{Na}_{\mathrm{v}} 1.5$ channel (Q1491H and G1481V) detected in 5- and 12-week-old infants diagnosed with a prolonged QT interval.

Methods: The $\mathrm{Na}_{\mathrm{v}} 1.5$ wild-type (WT) and the Q1491H and G1481V mutant channels were reproduced in vivo. WT or the mutant channels were co-transfected in HEK 293 cells with the beta 1 regulatory subunit. $\mathrm{Na}^{+}$currents were recorded using the whole-cell configuration of the patch-clamp technique.

Results: The Q1491H mutant channel exhibited a lower current density, a persistent $\mathrm{Na}^{+}$current, an enhanced window current due to a $+20-\mathrm{mV}$ shift of steady-state inactivation, a $+10-\mathrm{mV}$ shift of steady-state activation, a faster onset of slow inactivation, and a recovery from fast inactivation with fast and a slow time constants of recovery. The G1481V mutant channel exhibited an increase in current density and a +7 $\mathrm{mV}$ shift of steady-state inactivation. The observed defects are characteristic of gain-of-function mutations typical of LQT3.

Discussion and Conclusion: The 5- and 12-week-old infants displayed prolonged QT intervals. Our analyses of the Q1491H and G1481V mutations correlated with the clinical diagnosis. The observed biophysical dysfunctions associated with both mutations were most likely responsible for the sudden deaths of the two infants.

Key words: SCN5A, Na 1.5 , Voltage-gated sodium channels, Long QT syndrome, Variant, LQT3, Electrophysiology, Sudden cardiac death, Heart

\section{Introduction:}

Voltage-gated $\mathrm{Na}^{+}$channels are crucial for the amplitude and upstroke of cardiac action potentials (AP), which are important determinants for driving AP propagation and conduction velocity throughout the working myocardium ${ }^{1}$. Mutations in $S C N 5 A$, the gene that encodes $\mathrm{Na}_{\mathrm{v}} 1.5$, the predominant cardiac $\mathrm{Na}^{+}$ channel, have been implicated in rare familial forms of cardiac arrhythmias such as type 3 long QT syndrome (LQT3), Brugada syndrome $(B S)$, progressive cardiac conduction disorder ( $P C C D$ ), atrial fibrillation (AFib), and sudden infant death syndrome (SIDS). Another SCN5Amutation has recently been reported to be involved in dilated cardiomyopathy, a structural heart disease ${ }^{2,3}$. In addition to their role in changing gating characteristics, there is growing recognition that such mutations may also be associated with alterations in channel protein trafficking and expression levels. SCN5A gene is also the only gene to have a definitive evidence, for clinical validity of $\mathrm{BS}^{4}$.

Long QT syndrome (LQTS) is an inherited cardiac channelopathy that may lead to syncope and even sudden cardiac death as a result of polymorphic ventricular tachycardia known as torsade de pointes. LQTS manifests as a prolonged corrected QT (QTc) interval exceeding $450 \mathrm{~ms}$ on 12-lead electrocardiograms (ECG). To date, seventeen genes have been linked to inherited LQTS but only three have shown a definitive evidence as a genetic cause for typical LQTS. Those genes are KCNQ1, KCNH2 and SCN5A ${ }^{5}$.

LQT3 is caused by mutations in SCN5A genes mapped to chromosome 3q21-24, which encodes the $\alpha$ subunits of the cardiac $\mathrm{Na}^{+}$channel ${ }^{6,7}$. SCN5Amutations have been identified in about $10 \%$ of genotyped LQTS patients ${ }^{8}$. When a patient presents a LQTS, a genetic testing is used to identify the gene that cause the disease. If the gene is identified, then the clinician knows the type of LQTS (LQT1, LQT2 or LQT3) ${ }^{9}$. The development of arrhythmias is often associated with bradycardia during sleep or relaxation, when the QTc interval is prolonged. The most common mechanism for QT interval prolongation in LQT3 is due to a persistent or a late $\mathrm{Na}^{+}$current leading to an increase in AP plateau duration ${ }^{6}$. This can be a substrate for early after depolarizations (EADs), which can potentially trigger ventricular tachyarrhythmias. Most mutations linked to LQT3 are located in exons 23, 26, and 28, which encode the III-IV linker or inactivation gate, the voltage sensor domain, and the C-terminus of the $\mathrm{Na}^{+}$channel, all of which are involved in fast inactivation. These mutations impede the proper closure of the channel during this critical process, resulting in an increase in the number of channels that are unable to reach a stable inactivated state. This leads to a rise in persistent $\mathrm{Na}^{+}$currents unlike the normal inactivation of $\mathrm{WT} \mathrm{Na}^{+}$channels.

Although SIDS is the leading cause of death in the first year of life, its cause is still unknown. The only 
recommended preventive measure is to avoid placing infants on their stomachs or sides for sleep ${ }^{10}$. Several studies have linked SIDS to LQTS ${ }^{11}$ and have shown that $50 \%$ of infants who die of SIDS have a prolonged QTc interval and that a prolonged QTc interval over $440 \mathrm{~ms}$ in the first week of life increases the risk of SIDS by a factor of $41^{12,13}$.

We report here on two infants who presented with a prolonged QTc interval and who died suddenly, most likely after experiencing ventricular fibrillation. Although the 5- and 12-week-old infants were within the age range during which the incidence of SIDS peaks, their deaths were not attributed to SIDS. A sequencing analysis revealed a heterozygous G-to- $\mathrm{T}$ base substitution at position 4473 in exon 23 that resulted in a glutamate (E)-to-histidine $(\mathrm{H})$ substitution at residue 1491 and a heterozygous G-to- $\mathrm{T}$ base substitution at position 4442 in exon 23 that resulted in a glycine $(\mathrm{G})$-to-valine $(\mathrm{V})$ substitution at residue 1481. We recorded macroscopic $\mathrm{Na}^{+}$currents in transfected mammalian cells using the whole-cell configuration of the patch-clamp technique. The data revealed a marked shift of inactivation to more positive potentials, the presence of a persistent $\mathrm{Na}^{+}$current, and a large increase in the window current. All these characteristics point toward a gain-of-function due to the mutations.

\section{Methods}

\section{Institutional Committee on Human Research}

The study was conducted in accordance with the principles of the Declaration of Helsinki and the protocol was approved by the local ethics committee. The parents provided written informed consent.

\section{Cell cultures}

Human embryonic kidney 293 (HEK 293) cells were used. The cells were grown in high-glucose Dulbecco's modified Eagle's medium supplemented with $10 \%$ fetal bovine serum and $1 \%$ streptomycin at $37^{\circ} \mathrm{C}$ in a $5 \%$ $\mathrm{CO}_{2}$ atmosphere. The cells were transfected with the pcDNA3.1 vector containing either WT $\mathrm{Na}_{\mathrm{v}} 1.5 \mathrm{cDNA}$ (1? g) or $\mathrm{Na}_{\mathrm{v}} 1.5 / \mathrm{Q} 1491 \mathrm{H}$ and G1481V mutants with the pIRES2/EGFP vector containing $\beta 1$ subunit cDNA (1 ? g) in $10-\mathrm{cm}$ cell culture dishes using the calcium phosphate method as previously reported ${ }^{14}$.

\section{Whole-cell patch-clamp recordings}

$\mathrm{Na}^{+}$currents were recorded using low-resistance, fire-polished electrodes ([?] 1M $\Omega$ ) made from 8161 Corning borosilicate glass coated with HIPEC (Dow-Corning, Midland, MI, USA) to minimize electrode capacitance. An Axopatch 200 amplifier and pClamp software (Molecular Devices, Sunnyvale, CA, USA) were used to record $\mathrm{Na}^{+}$currents. The series resistance was compensated to $80 \%$ to minimize voltage-clamp errors. The cells were allowed to stabilize for $5 \mathrm{~min}$ after the whole-cell configuration was established. The membrane potential was held at $-140 \mathrm{mV}$ before the currents were recorded. Sodium Currents were filtered at $5 \mathrm{kHz}$ and digitized at $83.33 \mathrm{kHz}$. The liquid junction potential was not corrected. All the experiments were performed at room temperature $\left(22^{\circ} \mathrm{C}\right)$.

\section{Solutions}

The intracellular solution was composed of $35 \mathrm{mM} \mathrm{NaCl}, 105 \mathrm{mM}$ CsF, $10 \mathrm{mM}$ EGTA, and $10 \mathrm{mM}$ HEPES. The $\mathrm{pH}$ was adjusted to 7.3 with $2 \mathrm{M} \mathrm{CsOH}$. The first external solution (Low $\mathrm{Na}^{+}$) was composed of 35 $\mathrm{mM} \mathrm{NaCl}, 115 \mathrm{NMDG}, 2 \mathrm{mM} \mathrm{KCl}, 1.5 \mathrm{mM} \mathrm{CaCl}_{2}, 1 \mathrm{mM} \mathrm{MgCl}_{2}, 10 \mathrm{mM}$ glucose, and $10 \mathrm{mM}$ HEPES. The $\mathrm{pH}$ was adjusted to 7.4 with $2 \mathrm{M} \mathrm{HCl}$. The second external solution (Full $\mathrm{Na}^{+}$) used to record persistent $\mathrm{Na}^{+}$currents was composed of $150 \mathrm{mM} \mathrm{NaCl}, 2 \mathrm{mM} \mathrm{KCl}, 1.5 \mathrm{mM} \mathrm{CaCl}_{2}, 1 \mathrm{mM} \mathrm{MgCl}, 10 \mathrm{mM}$ glucose, and $10 \mathrm{mM}$ HEPES. The $\mathrm{pH}$ was adjusted to 7.4 with $2 \mathrm{M} \mathrm{HCl}$. TTX (LATOXAN, Portes-lès-Valence, France) was diluted to 75 ?M in $2 \%$ acetic acid.

\section{Data analysis}

The slope factor $(\mathrm{K})$ and the midpoint $\left(\mathrm{V}_{1 / 2}\right)$ for activation and inactivation were calculated using standard Boltzmann functions: $1 /\left(1+\exp \left(\left(\mathrm{V}_{1 / 2 \text { activation }}-\mathrm{V}\right) / \mathrm{k}_{\text {activation }}\right)\right)$ for activation and $(1-\mathrm{C}) /(1+$ $\left.\left.\exp \left(\left(\mathrm{V}-\mathrm{V}_{1 / 2 \text { inactivation }}\right) / \mathrm{k}_{\text {inactivation }}\right)\right)+\mathrm{C}\right)$ for inactivation. $\mathrm{V}$ is the voltage. The window current 
was obtained using equation 1: $\left(1 /\left(1+\exp \left(\left(\mathrm{V}_{1 / 2 \text { activation }}-\mathrm{V}\right) / \mathrm{k}_{\text {activation }}\right)\right) \times((1-\mathrm{C}) /(1+\exp ((\mathrm{V}-\right.$ $\left.\left.\left.\left.\mathrm{V}_{1 / 2 \text { inactivation }}\right) / \mathrm{k}_{\text {inactivation }}\right)\right)+\mathrm{C}\right) \times 100$, which is the probability of having the channel in the open state.

Statistical analysis

Results are expressed as means +- S.E.M. Statistical comparisons were performed using a one-way ANOVA in GraphPad Prism for statistical comparisons. Differences were considered significant at $\mathrm{P}<0.05$.

\section{Results}

\section{Available clinical data and identification of the SCN5A mutations}

Patient \#1 was an infant with a QTc of $700 \mathrm{~ms}$ and a 2:1 atrioventricular block (AVB) at birth (Fig 1A). The 2:1 AVB was detected in utero at a gestational age of $31 \mathrm{WA}$. This infant received $2 \mathrm{mg} / \mathrm{kg}$ of propranolol, and a pacemaker was implanted a few days after birth. He experienced salvos of torsades de pointes. Flecainide and mexiletine were unsuccessful in preventing torsades. The patient died at 3 months of age after experiencing ventricular fibrillation.

Patient \#2 had a QTc of $600 \mathrm{~ms}$ and a 2:1 AVB block at birth. The 2:1 AVB was detected in utero at a gestational age of $25 \mathrm{WA}$. A pacemaker was implanted a few days after birth, and $2 \mathrm{mg} / \mathrm{kg}$ of propranolol was started. The infant experienced several episodes or torsades de pointes and died at 1 month of age after experiencing ventricular fibrillation.

The genomic DNAs of the two infants were screened using specific primers for variant changes in several genes associated with arrhythmia syndrome (see Supplemental Table 1), in particular in all 28 SCN5A exons. Molecular screening by automatic sequencing analysis of the genomic DNAs revealed two novel mutations in the SCN5A gene (G1481V and Q1491H), namely, c. $4473 \mathrm{G}>\mathrm{T}$, which resulted in a Glu1491His substitution, and c. $4442 \mathrm{G}>\mathrm{T}$, which resulted in a Gly1481Val substitution (Fig 1B). No mutations were detected in other genes. Applying the American College of Medical Genetics and Genomics (ACMG) guidelines ${ }^{15}$, revealed unclear initial results. This is illustrated and confirmed by Varsome (https://varsome.com) and CardioClassifier (https://www.cardioclassifier.org) which respectively returned both variations as likely pathogenic (class 4) or uncertain significance (class 3). This initial variant classification consequently precluded their use in future presymptomatic diagnosis. This warranted further detailed biophysical characterization (see below) The two substitutions are highly conserved in the $\mathrm{Na}+$ channels of many species and are located in the III-IV linker, which plays a role in channel inactivation in all voltage-gated $\mathrm{Na}^{+}$channels. It was hypothesized that these two mutations were responsible for the sudden death events. Family screening documented normal phenotypes in both parents of the two infants.

\section{Biophysical characteristics of $\mathrm{Na}_{\mathrm{v}} 1.5 / \mathrm{Q} 1491 \mathrm{H}$ and $\mathrm{Na}_{\mathrm{v}} 1.5 / \mathrm{G} 1481 \mathrm{~V}$}

Currents were elicited by sequential depolarizing steps of the cell membrane from $-100 \mathrm{mV}$ to $+30 \mathrm{mV}$ in 5-mV increments (Fig. 2A) with the Low $\mathrm{Na}^{+}$external solution (see Methods). The I-V curves were constructed by measuring the peak of each $\mathrm{Na}^{+}$current and were normalized to the membrane capacitance to obtain current densities ( $\mathrm{pA} / \mathrm{pF}$ ). The current density of the Q1491H mutant channel was lower than that of the WT channel while the current density of the G1481V mutant channel was higher than that of the WT channel. These results suggest that the expression levels of the Q1491H and G1481V mutant channels were different than that of the WT channel. The potential of the maximum peak current amplitude of the Q1491H channel was shifted toward more positive potentials compared to the WT channel (Fig. 2B) . To study the activation and inactivation parameters, we first calculated the G-V curves (steady-state activation), which were fitted with a standard Boltzmann function. The $\mathrm{V}_{1 / 2}$ of the $\mathrm{Q} 1491 \mathrm{H}$ mutant channel was positively shifted by $9 \mathrm{mV}$, with significant differences in $\mathrm{k}$ values. There was no difference between the $\mathrm{V}_{1 / 2}$ values of the WT channel and the G1481V mutant channel (Fig. 3A and Table 1).

Voltage-dependent steady-state inactivation was assessed by applying 500-ms pre-pulses ranging from -140 $\mathrm{mV}$ to $-30 \mathrm{mV}$ to allow the channels to enter the inactivated state followed by a test pulse at $-30 \mathrm{mV}$ to assess the number of functional channels. The current amplitude of the test pulse was normalized to the 
maximum current recorded during the pre-pulse and was plotted versus the voltage of the pre-pulse to obtain the voltage-dependent inactivation curve, which was then fitted to a standard Boltzmann function. $\mathrm{V}_{1 / 2}$ and $\mathrm{k}$ were generated by fitting each data set with a standard Boltzmann function (see values in Table 1 ). A $+20-\mathrm{mV}$ shift for $\mathrm{Q} 1491 \mathrm{H}$ and a $+7-\mathrm{mV}$ shift for G1481V were observed, but $\mathrm{k}$ was not significantly affected (Fig. 3A) .

Window currents were determined and were used to assess the open probability. The total areas were 1.4 and 11-fold larger, respectively, for G1481V and Q1491H than for the WT channel and were shifted to more positive voltages (Fig. 3B) .

Slow inactivation was measured using a two-pulse protocol, with an initial conditioning pulse (pre-pulse) and a final test pulse. The current was normalized to the current amplitude during the pre-pulse. There was no difference between the G1481V and WT channels in terms of the kinetics of entry into slow inactivation. However, the Q1491H mutant channel entered into slow activation faster than the WT channel(Fig. 4A) . Recovery from slow inactivation was measured using a two-pulse protocol. The channels were first inactivated by a $500-\mathrm{ms}$ pre-pulse and were then allowed to recover from inactivation. The peak currents were normalized to the maximum peak current. The Q1491H mutant exhibited a complex recovery with two (fast and slow) time constants(Table 1). The G1481V mutant recovered from slow activation more quickly than the WT channel (Fig. 4B). The currents of the mutant channels (Q1491H and G1481V) did not decrease during closed-state inactivation. Since the WT channel only exhibited a $\tau_{\text {fast }}$ time constant, $\tau_{\text {slow }}$ is not present in Table 1 . Closed-state inactivation was assessed using a two-pulse protocol. The currents were normalized to the first pulse. Neither mutated channel was affected, unlike the WT channel, which showed closed-state inactivation (Fig. 4C) .

We recorded the frequency-dependence of the $\mathrm{Na}^{+}$currents in order to determine the channels that enter the inactivated state by applying a series of 50 depolarizing pulses at $-40 \mathrm{mV}$. To evaluate current inhibition during rapid pulsing, the channels were pulsed 50 times at $2 \mathrm{~Hz}, 5 \mathrm{~Hz}$, and $10 \mathrm{~Hz}$. There was a significant difference between the WT channel and the Q1491H mutant channel at $10 \mathrm{~Hz}$, with the mutant channel displaying a reduced current during rapid pulsing. There was no difference between the G1481V mutant channel and the WT channel (Fig. 5) .

\section{Persistent $\mathrm{Na}^{+}$currents in cells transfected with the Q1491H mutant channel}

Persistent non-inactivating $\mathrm{Na}^{+}$currents were recorded using a 400-ms depolarization pulse from $-140 \mathrm{mV}$ to $-30 \mathrm{mV}$ (see protocol inset) and were normalized to the peak. The current was inhibited using 25 ?M tetrodotoxin (TTX). Only the Q1491H mutant channel displayed a significant decrease in current (2.66 +0.29 to $1.25+-0.22$ ), indicating the presence of a persistent current (Fig. 6) . TTX had no effect on the G1481V mutant and WT currents, which is indicative of a non-specific leak.

\section{Discussion}

Q1491H and G1481V are SCN5A heterozygous mutations identified in two infants who died from long QT (Q1491H $700 \mathrm{~ms}$ AVB 2:1; G1481V $600 \mathrm{~ms}$ AVB 2:1) at 5 (G1481V) and 12 (Q1491H) weeks of age, respectively. The mutations were located in the $\mathrm{Na}_{\mathrm{v}} 1.5$ intracellular loop between domain III and IV near the IFM motif (Fig 1), which is involved in fast inactivation ${ }^{16}$. Such mutations in the inactivation gate are often associated with changes in inactivation of voltage-gated $\mathrm{Na}^{+}$channels. Both mutations were identified in clinical practice. SCN5A gene is known as a clinically actionable gene (https://search.clinicalgenome.org). The variants pathogenic status is thus critical to inform appropriately the family and their use in presymptomatic diagnosis.

The Q1491H mutation was located four amino acids downstream from the IFM motif. Glutamine has a polar uncharged side chain whereas histidine has electrically charged side chains. A biophysical characterization revealed that this mutation has a marked effect on the normal function of the Nav1.5 channel. We observed a loss-of-function for peak current density that could be due to a trafficking defect. We also observed a loss-of-function for recovery time (a slow time constant: $56.01+-8.73 \%$, Table $\mathbf{1}$ ), which led to a decreased 
inward $\mathrm{Na}^{+}$current and a longer recovery from inactivation time. An increase in the window current may lead to an increase in $\mathrm{Na}^{+}$influx, which can increase the risk of fatal ventricular arrhythmias. This was correlated with the depolarized shift of steady state inactivation $(+20.27 \mathrm{mV})$ and activation $(+9.36 \mathrm{mV})$ that drove channel activity to more positive potentials. We also showed that the Q1491H mutant channel displays a persistent current that is inhibited by 25 ?M TTX (Fig. 6) and ranolazine (Fig. 7), which is characteristic of LQT3. This mutation also displayed pronounced frequency-dependent inactivation at 10 $\mathrm{Hz}$, suggesting that the $\mathrm{Na}_{\mathrm{v}} 1.5 / \mathrm{Q} 1491 \mathrm{H}$ channel has an unstable inactivated state. These results provide a rationale for linking this mutation and the clinical phenotype, especially for the long QTc interval (700 ms) measured in the infant prior to its sudden death.

The G1481V mutation was located four amino acids upstream from the IFM motif. Since valine and glycine are both uncharged, this may explain the more modest effect on the function of the Nav1.5/G1481V channel. The G1481V mutation resulted in an increase in the current density(Fig. 2) as well as a $+7.03-\mathrm{mV}$ depolarized shift in inactivation, which increased by 1.4-fold the window current(Fig. 3) . This mutation did result in a significant increase in persistent current; however the use of other expression system such as iPSCs is warranted. Furthermore, the recovery from the inactivation was also faster indicating that sodium channel will recover faster from inactivation All these changes represent a gain of function of G1481V mutant sodium channels.

The biophysical characterization of both Q1491H and G1481V SCN5Amutations thus revealed a functional defect. The manual patch-clamp is not validated as a well-established method in a variant classification perspective $^{17,18}$. Considering the clear biophysical defects, a "Pathogenic Strong 3" (PS3) criterion in the ACMG classification process. Interestingly, both variants were re-classified as either likely pathogenic (class 4) or pathogenic (class 5), indicating that with the support of the biophysical characterization, both SNC5A variants can now be used for pre-symptomatic diagnosis.

Interestingly, although the patient with the G1481V mutation presented a long QTc interval of $600 \mathrm{~ms}$, the increase in the persistent current was not statistically significant. In addition, there was no significant difference in the time constants of inactivation for all conditions (data not shown).

The III-IV linker acts as a lid in which the inactivation particle isoleucine-phelynalanine-methionine (IFM) amino acid trio occludes the inner pore by binding to the docking site like a latch. We used the recently crystalized rat Nav1.5 to visualize the position of the two residues and their interacting sites ${ }^{19}$. The IFM motif is highly conserved in all $\mathrm{Na}^{+}$channels. The sites of the $\mathrm{Q}$ and $\mathrm{G}$ mutations investigated in the present study were located up- and downstream, respectively, from the IFM. They are highly conserved in several $\mathrm{Na}^{+}$ channels, underlining their importance during the process of inactivation. The structure revealed that Q1493 residue interacts with N1661 (N1659 in hNav1.5) and is one of the key residues for optimum IFM docking. It has been shown earlier that mutating N1661 to alanine abolishes fast inactivation ${ }^{20}$. The recently published rat Nav1.5 structure showed that Q1493 at the C-terminal of the DIII-DIV linker (Q1491 in hNav1.5) forms a strong hydrogen bond with N1661 (Fig. 8) . Q1491H mutation may lead to a weaker interaction with N1659 because of histidine shorter imidazole side chain. This will impair fast inactivation and lead to a gain of function effect. This may explain the appearance of persistent current and the rightward shift of the steady-state inactivation curve of Q1491H mutant. This could impede this interaction and contribute to change in pore closure and the slowing of slow inactivation. On the other hand, G1481 which is located at the tight turn of DIII S6 and the beginning DIII-DIV linker is important for keeping the flexibility of movement of the IFM motif. A valine substitution, with its bulky side chain, at this position will lead to less flexibility of the IFM motif movement. This structural insight explain the mild slow fast inactivation kinetics of G1481V mutant compared to wild type channel. However, the effects of this mutation is expected to be less drastic compared to Q1491H mutation. This is manifested by the little effects of G1481V mutation on channel function

Many SCN5A mutations, especially in the III-IV linker and the voltage sensor domain, have been linked to $\mathrm{BrS}$ and LQT3. The difference between BrS and LQT3 is that BrS appears with age ${ }^{21}$. The first mutation to be associated with LQT3 was a three-amino-acid deletion $\left(\mathrm{Lys}_{1505}-\mathrm{Pro}_{1506}-\mathrm{Gln}_{1507}\right.$, [?]KPQ). This mutation 
shares the same deletion (Q1507) as the delQKP deletion at position 1507-1509. Both are located in the IIIIV linker region and are associated with a gain-of-function and a late persistent $\mathrm{Na}^{+}$current ${ }^{22,23}$. This region is a hotspot of mutations that induce inactivation disturbances, including a depolarized shift in inactivation that enhances the window current, as is the case for Q1491H and G1481V. This same effect was observed with F1486del, which involves a deletion of a phenylalanine on the IFM motif ${ }^{24}$. This mutation alters lidocaine sensitivity, which is a local anesthetic that blocks late currents associated with LQT3 mutations. Although IFM/QQQ mutants also display an alteration in lidocaine sensitivity ${ }^{25}$, this effect is not seen if the phenylalanine $(\mathrm{F})$ is replaced by a glutamine $(\mathrm{Q})^{26}$. Other mutations have been shown to induce a long QTc interval, including $\mathrm{N} 1774 \mathrm{H}$, which is located in the C-terminal ${ }^{27}$. Like the Q1491H mutations, N1774H results in a loss-of-function in peak current density and an increase in the late current. The $\mathrm{N} 1774 \mathrm{H}$ mutation was detected in a 19-year-old woman. The S1333Y mutation on the S4-5 linker results in an enhanced window current and a persistent current. The S1333Y mutation was detected in a 25-day-old infant ${ }^{28}$. Although all these mutations lead to LQT3, they are located in different parts of the $\mathrm{Na}_{\mathrm{v}} 1.5$ channel that all play a role in inactivation. The ineffectiveness of mexiletine and flecainide in case 1 and propranolol in case 2 , is not very clear especially that these drugs all have some sodium channels blocking properties. The possibility of Inter-individual pharmacokinetic and pharmacodynamic differences could explain the non-uniform clinical response to these drugs.

\section{Conclusion}

The Q1491H and G1481V mutations are both located near the IFM motif, which is involved in fast inactivation. These mutations changed the inactivation state, shifting it to a more positive voltage. They also increased the window current, which can result in heart malfunctions such as LQT3. Full biophysical characterization constitutes a strong benefit, providing useful supplemental criterion in the variant classification procedure. These two infants may have benefited from class 1 antiarrhythmic drug therapy, but the lack of effect these drugs is not well understood and warrants further study.

\section{Acknowledgements:}

This research was supported by the Canadian Institutes of Health Research (grants MOP-111072 and MOP130373 to MC) and Association Francaise contre les Myopathies - Telethon (grant AFM19962 to MC).

\section{References}

1. Coraboeuf E, Deroubaix E, Coulombe A. Effect of tetrodotoxin on action potentials of the conducting system in the dog heart. Am J Physiol - Hear Circ Physiol. 1979;5(4).

2. Moreau A, Gosselin-Badaroudine P, Delemotte L, Klein ML, Chahine M. Gating pore currents are defects in common with two Nav1.5 mutations in patients with mixed arrhythmias and dilated cardiomyopathy. J Gen Physiol. 2015;145(2):93-106.

3. Gosselin-Badaroudine P, Keller DI, Huang H, et al. A proton leak current through the cardiac sodium channel is linked to mixed arrhythmia and the dilated cardiomyopathy phenotype. PLoS One. 2012;7(5).

4. Hosseini SM, Kim R, Udupa S, et al. Reappraisal of Reported Genes for Sudden Arrhythmic Death. Circulation. 2018;138(12):1195-1205.

5. Adler A, Novelli V, Amin AS, et al. An International, Multicentered, Evidence-Based Reappraisal of Genes Reported to Cause Congenital Long QT Syndrome. Circulation. 2020;141(6):418-428.

6. George AL, Varkony TA, Drabkin HA, et al. Assignment of the human heart tetrodotoxin-resistant voltage-gated $\mathrm{Na}+$ channel $\alpha$-subunit gene (SCN5A) to band 3p21. Cytogenet Genome Res. 1995;68(12):67-70. doi:7956363

7. Bennett, Paul B.; Yazawa, Kazuto; Makita, Naomasa; George AL. Molecular mechanisms for an inherited cardiac arrhythmia.pdf. 
8. Tan BH, Pundi KN, Van Norstrand DW, et al. Sudden infant death syndrome-associated mutations in the sodium channel beta subunits. Hear Rhythm. 2010;7(6):771-778.

9. Gollob MH, Blier L, Brugada R, et al. Recommendations for the Use of Genetic Testing in the Clinical Evaluation of Inherited Cardiac Arrhythmias Associated with Sudden Cardiac Death: Canadian Cardiovascular Society/Canadian Heart Rhythm Society Joint Position Paper. Can J Cardiol. 2011;27(2):232-245.

10. Dwyer T, Ponsonby AL, Blizzard L, Newman NM, Cochrane JA. The contribution of changes in the prevalence of prone sleeping position to the decline in sudden infant death syndrome in tasmania. Obstet Gynecol Surv. 1995;50(10):704-705.

11. VILKE GM, CHAN TC. A Molecular Link Between The Sudden Infant Death Syndrome And The Long-QT Syndrome. Prehospital Emerg Care. 2002;6(2):259-259.

12. England TN. Prolongation Of The QT Interval And The Sudden Infant Death Syndrome. Published online 1998:1709-1714.

13. Schwartz PJ, Stramba-Badiale M. Repolarization abnormalities in the newborn. J Cardiovasc Pharmacol. 2010;55(6):539-543.

14. Huang H, Priori SG, Napolitano C, O'Leary ME, Chahine M. Y1767C, a novel SCN5A mutation, induces a persistent $\mathrm{Na}+$ current and potentiates ranolazine inhibition of Nav1.5 channels. Am J Physiol - Hear Circ Physiol. 2011;300(1):288-299.

15. Richards S, Aziz N, Bale S, et al. Standards and guidelines for the interpretation of sequence variants: a joint consensus recommendation of the American College of Medical Genetics and Genomics and the Association for Molecular Pathology. Genet Med. 2015;17(5):405-423.

16. Chahine M, Deschene I, Chen LQ, Kallen RG. Electrophysiological characteristics of cloned skeletal and cardiac muscle sodium channels. Am J Physiol - Hear Circ Physiol. 1996;271(2 40-2):498-506.

17. Kanavy DM, McNulty SM, Jairath MK, et al. Comparative analysis of functional assay evidence use by ClinGen Variant Curation Expert Panels. Genome Med. 2019;11(1):77.

18. Brnich SE, Abou Tayoun AN, Couch FJ, et al. Recommendations for application of the functional evidence PS3/BS3 criterion using the ACMG/AMP sequence variant interpretation framework. Genome Med. 2020;12(1):3.

19. Jiang D, Shi H, Tonggu L, et al. Structure of the Cardiac Sodium Channel. Cell. 2020;180(1):122134.e10.

20. McPhee JC, Ragsdale DS, Scheuer T, Catterall WA. A Critical Role for the S4-S5 Intracellular Loop in Domain IV of the Sodium Channel $\alpha$-Subunit in Fast Inactivation. J Biol Chem. 1998;273(2):1121-1129.

21. Beaufort-Krol GCM, Van Den Berg MP, Wilde AAM, et al. Developmental aspects of long QT syndrome type 3 and brugada syndrome on the basis of a single SCN5A mutation in childhood. J Am Coll Cardiol. $2005 ; 46(2): 331-337$.

22. Keller DI, Acharfi S, Delacrétaz E, et al. A novel mutation in SCN5A, delQKP 1507-1509, causing long QT syndrome: Role of Q1507 residue in sodium channel inactivation. J Mol Cell Cardiol. 2003;35(12):15131521.

23. Wang Q, Shen J, Splawski I, et al. SCN5A mutations associated with an inherited cardiac arrhythmia, long QT syndrome. Cell. 1995;80(5):805-811.

24. Song W, Xiao Y, Chen H, et al. The human Nav1.5 F1486 deletion associated with long QT syndrome leads to impaired sodium channel inactivation and reduced lidocaine sensitivity. J Physiol. 2012;590(20):51235139 . 
25. Bennett PB, Valenzuela C, Chen LQ, Kallen RG. On the molecular nature of the lidocaine receptor of cardiac $\mathrm{Na}+$ channels modification of block by alterations in the $\alpha$-subunit III-IV interdomain. Circ Res. 1995;77(3):584-592.

26. Balser JR, Nuss HB, Romashko DN, Marban E, Tomaselli GF. Functional consequences of lidocaine binding to slow-inactivated sodium channels. J Gen Physiol. 1996;107(5):643-658.

27. Neubauer J, Wang Z, Rougier J, et al. Functional characterization of a novel SCN5A variant associated with long QT syndrome and sudden cardiac death. Int J Legal Med. Published online 2019.

28. Huang H, Millat G, Rodriguez-Lafrasse C, et al. Biophysical characterization of a new SCN5A mutation S1333Y in a SIDS infant linked to long QT syndrome. FEBS Lett. 2009;583(5):890-896.

\section{Figure legends}

Figure 1: (A) Standard ECG recorded soon after birth. Paper speed $25 \mathrm{~mm} / \mathrm{s} ; 10 \mathrm{~mm} / 1 \mathrm{mV}$. The QT interval was $720 \mathrm{~ms}$, and the QRS was $60 \mathrm{~ms}$ and 2:1 AVB developed due to an extremely prolonged QT interval. (B) Sequence analysis of the G1481V and Q1491H mutations. (C) The Q1491H mutation resulted from a G- to - T substitution at position 4473, leading to a glutamine (Q)-to-histidine (H) substitution at residue 1491. The G1481V mutation resulted from a G- to $-\mathrm{T}$ substitution at position 4442 , leading to a glycine $(\mathrm{G})$-to-valine $(\mathrm{V})$ substitution at residue 1481. (D) Schematic representation of the four domains of the $\alpha$-subunit of the $\mathrm{Na}_{\mathrm{v}} 1.5$ channel showing the locations of the Q1491 and G1481V mutations. DEKA represents the selectivity filter of the channel. The Q1491H mutation caused a glutamine-to-histidine substitution four amino acids downstream from the IFM motif. The G1481V mutation caused a glycine-to-valine substitution four amino acids upstream from the IFM motif.

Figure 2 : Analysis of whole-cell $\mathrm{Na}^{+}$currents recorded from HEK 293 cells expressing $\mathrm{Na}_{\mathrm{v}} 1.5 \mathrm{WT}, \mathrm{Q} 1491 \mathrm{H}$, and G1481V. (A) Example of raw traces from $\mathrm{Na}_{\mathrm{v}} 1.5 / \mathrm{WT}, \mathrm{Q} 1491 \mathrm{H}$, and G1481V were obtained using depolarizing pulses from $-100 \mathrm{mV}$ to $+30 \mathrm{mV}$ in 5 -mV increments. (B) Current-voltage relationship of $\mathrm{WT} *(\mathrm{n}=9)$, Q1491H [?] $(\mathrm{n}=9)$, and G1481V ${ }^{n}=$ 9).Thecurrentamplitudewasnormalizedtothemembranecapacitancetoobtainthecurrentdensity $(p A / p F)$.

Figure 3: Gating properties of steady-state activation and inactivation and window currents. (A) Voltagedependence of steady-state activation and inactivation in WT $(*$, activation, $\mathrm{n}=9$, and inactivation, $\mathrm{n}=9)$, Q1491H ([?], activation, $\mathrm{n}=9$, and inactivation, $\mathrm{n}=7$ ), and G1481V ('activation, $n=9$, andinactivation, $n=$ 7).Theinactivatedcurrentsweregeneratedusingtheprotocoldescribedintheinsets, andtheactivatedcurrentswereobtainedfron

Figure 4: The gating properties of slow inactivation, recovery from slow inactivation, and closed-state inactivation. (A) Slow inactivation in WT (*, $\mathrm{n}=6)$, Q1491H ([?], $\mathrm{n}=6)$, and G1481V (' $n=6)$. Thetwo pulseprotocoldescribedintheinsetwasusedtogeneratethecurrents.(B)Timecoursesofrecovery fromslowinactivationinWT(, 7), Q1491H([?], $n=8)$, andG1481V('n=7).Closed - stateinactivationinWT $(*, n=7), Q 1491 H([?], n=$ $8)$, andG1481V('n=7).

Figure 5: Frequency dependence of WT $(*, \quad n=8), \quad$ Q1491H ([?], n=8), and G1481V $\left({ }^{\prime} n=\right.$ 8).Currentswereevaluatedat $(A) 2,(B) 5$, and $(C) 10$ Hz.Fiftypulseswereappliedat $-40 \mathrm{mV}$ fromaholdingpotentialof--140mV.Peakcurrentswerenormalizedtothe firstpeakcurrentandwereplottedver susthe

Figure 6: Persistent $\mathrm{Na}^{+}$currents in WT, Q1491H, and G1481V inhibited by TTX. Recordings were performed using a $-30-\mathrm{mV}$ depolarizing pulse (see protocol in inset). (A) Currents recorded without TTX (left) and with 25 ?M TTX (right). (B) Currents normalized to the maximum current, without TTX (left) and with 25 ?M TTX (right). (C) Histograms of persistent $\mathrm{Na}^{+}$currents. The persistent currents accounted for $0.58 \pm 0.10$ of the peak current amplitude for WT without TTX $(\mathrm{n}=6), 2.66 \pm 0.29$ for Q1491H without TTX ( $\mathrm{n}=13), 1.25 \pm 0.22$ for Q1491H with 25 ?M TTX ( $=13)$, and $1.33+-0.35$ for G1481V without TTX $(\mathrm{n}=11)$ at $-30 \mathrm{mV}(* * \mathrm{P}<0.01, * * * \mathrm{P}<0.001)$.

Figure 7: Effects of ranolazine on persistent sodium current. (A) Persistent $\mathrm{Na}^{+}$current traces using the same protocol as shown in Fig. 6 showing the effect of $100 \mu \mathrm{M}$ ranolazine. (B) Bar graph of 
persistent $\mathrm{Na}^{+}$currents inhibition by ranolazine $\left({ }^{*} \mathrm{P}<0.05\right)$.

Figure 8: Open state structure of the human Nav1.5 sodium channel.

(A) Top view of the Cryo-EM structure of rat Nav1.5 channel showing the IFM motif in red spheres. (B) Bottom view of the Cryo-EM structure of the rat Nav1.5 channel showing the IFM motif in red spheres.

(C) Close-up view of the IFM motif flanked by G1481 on the N-terminal and Q1491 on the C-terminal. A strong hydrogen bond between N1661 and Q1491 (2.9A) ensures the proper docking of IFM motif.

Table 1: Biophysical properties of the Nav1.5 WT and mutant channels

$\mathrm{Na}_{\mathrm{v}}$ 1.5/WT $\quad \mathrm{Na}_{\mathrm{v}} 1.5 / \mathrm{O} 1491 \mathrm{H} \quad \mathrm{Na}_{\mathrm{v}} 1.5 / \mathrm{G} 1481 \mathrm{~V}$

\begin{tabular}{|c|c|c|c|}
\hline Current Density & & & \\
\hline $\begin{array}{l}\text { Maximum Peak } \\
(\mathrm{pA} / \mathrm{pF})\end{array}$ & $-235.8 \pm 39.8(n=9)$ & $-49.2 \pm 7.3(n=9)$ & $-391.2 \pm 48.3(n=9)$ \\
\hline \multicolumn{4}{|l|}{$\begin{array}{l}\text { Steady-state } \\
\text { inactivation }\end{array}$} \\
\hline $\mathrm{V} 1 / 2(\mathrm{mV})$ & $-89.6 \pm 1.6(n=9)$ & $-69.4 \pm 1.5^{* * * *}(n=7)$ & $-82.6 \pm 2.3^{*}(n=7)$ \\
\hline $\mathrm{K}(\mathrm{mV})$ & $-5.1 \pm 0.1(n=9)$ & $-4.5 \pm 0.3(n=7)$ & $-4.6 \pm 0.2(n=7)$ \\
\hline \multicolumn{4}{|l|}{$\begin{array}{l}\text { Steady-state } \\
\text { activation }\end{array}$} \\
\hline $\mathrm{V} 1 / 2(\mathrm{mV})$ & $-41.4 \pm 2.5(n=9)$ & $-32.0 \pm 1.5^{* *}(n=9)$ & $-42.2 \pm 1.2(n=9)$ \\
\hline $\mathrm{K}(\mathrm{mV})$ & $7.642 \pm 1.0(n=9)$ & $14.2 \pm 1.3^{* * *}(\mathrm{n}=9)$ & $8.0 \pm 0.6(n=9)$ \\
\hline
\end{tabular}

Recovery from Slow

inactivation

\begin{tabular}{lccc}
\hline$\tau_{\text {fast }}(\mathrm{ms})$ & $4.2 \pm 0.6(\mathrm{n}=7)$ & $0.8 \pm 0.1^{* * * *}(\mathrm{n}=8)$ & $1.9 \pm 0.2^{* * *}(\mathrm{n}=7)$ \\
\hline$\tau_{\text {slow }}(\mathrm{ms})$ & $/$ & $55.9 \pm 5.0(\mathrm{n}=8)$ & $88.2 \pm 12.2(\mathrm{n}=8)$ \\
\hline $\mathrm{A}_{\text {fast }}$ & $91.0 \pm 1.1 \%(\mathrm{n}=5)$ & $56.0 \pm 8.7 \%^{* *}(\mathrm{n}=8)$ & $83.2 \pm 4.5 \%(\mathrm{n}=7)$ \\
\hline $\mathrm{A}_{\text {slow }}$ & $9.0 \pm 1.1 \%(\mathrm{n}=5)$ & $44.0 \pm 8.7 \%^{* *}(\mathrm{n}=8)$ & $16.8 \pm 4.5 \%(\mathrm{n}=7)$ \\
\hline
\end{tabular}

\begin{tabular}{llll}
\hline $\begin{array}{l}\text { Onset of slow } \\
\text { inactivation }\end{array}$ & & & \\
\hline Plateau & $0.9 \pm 0.1(n=6)$ & $0.7 \pm 0.1^{* * *}(n=6)$ & $0.8 \pm 0.1(n=5)$ \\
\hline & & & \\
\hline $\begin{array}{l}\text { Closed State of } \\
\text { inactivation }\end{array}$ & & & \\
\hline Plateau & $0.8 \pm 0.1(n=8)$ & $1.1 \pm 0.1^{* * * *}(n=10)$ & $1.0 \pm 0.1^{* * *}(n=7)$
\end{tabular}

$V_{1 / 2}=$ midpoint for activation or inactivation, $K v=$ slow factor for activation or inactivation, $s=$ time 
constant, and $A=$ fraction of recovery component.

${ }^{*} P<0.05,{ }^{* *} P<0.01,{ }^{* * *} P<0.001,{ }^{* * * *} P<0.0001$
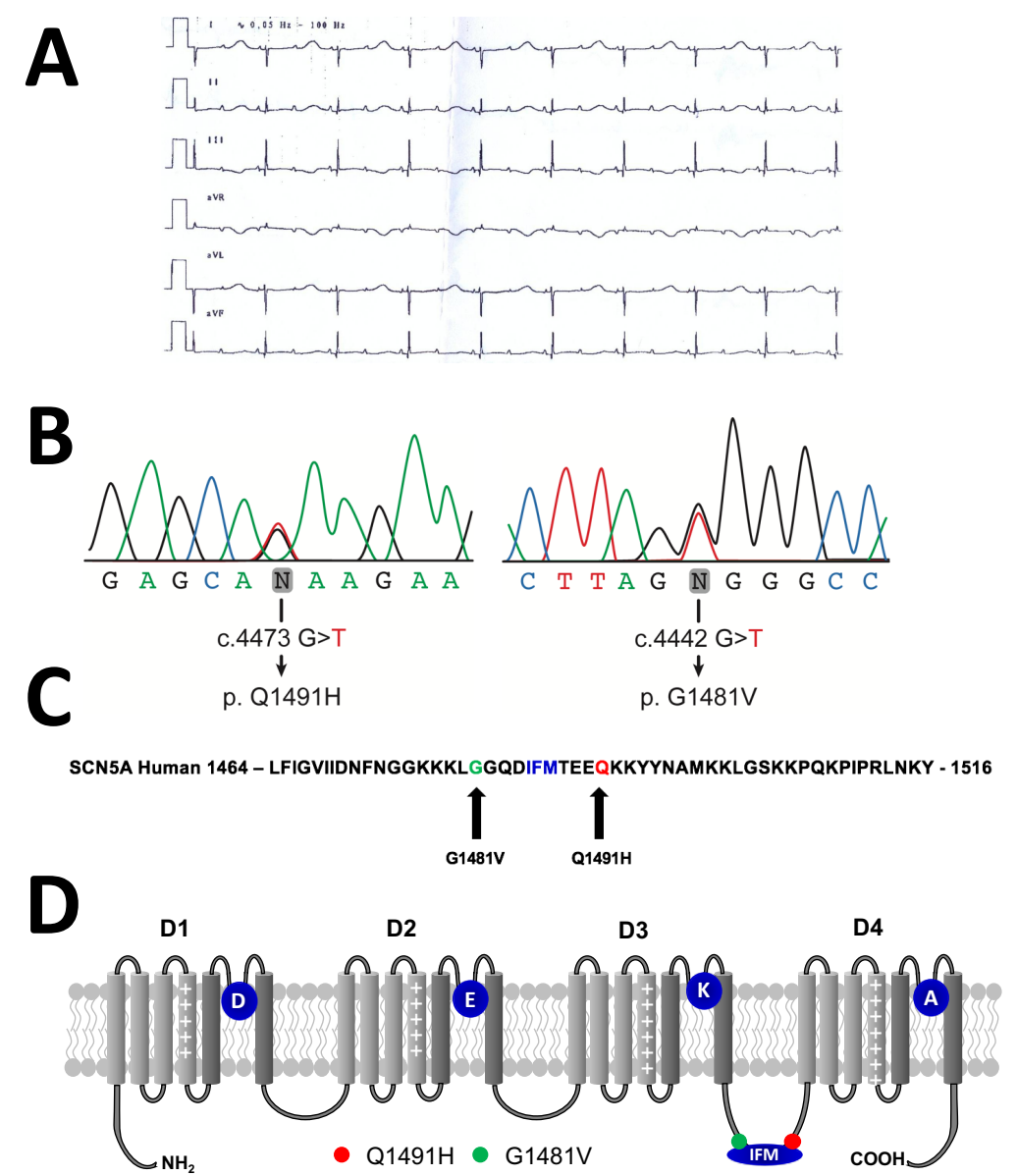


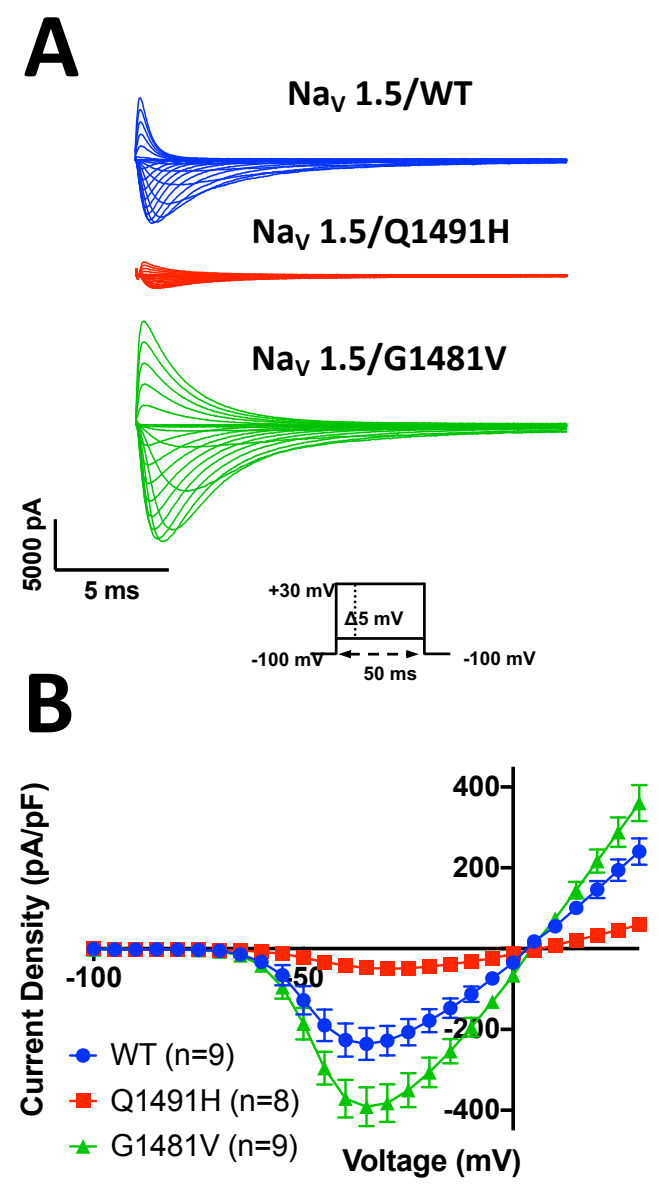




\section{A}
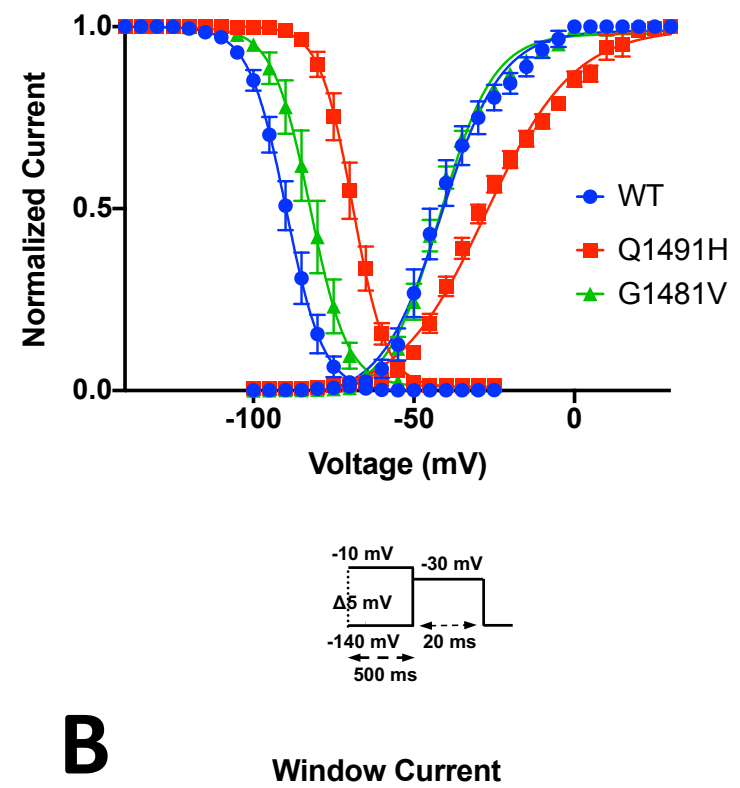

Window Current

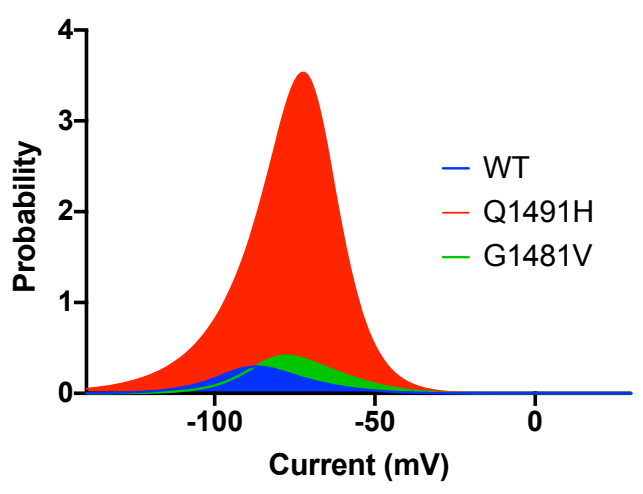



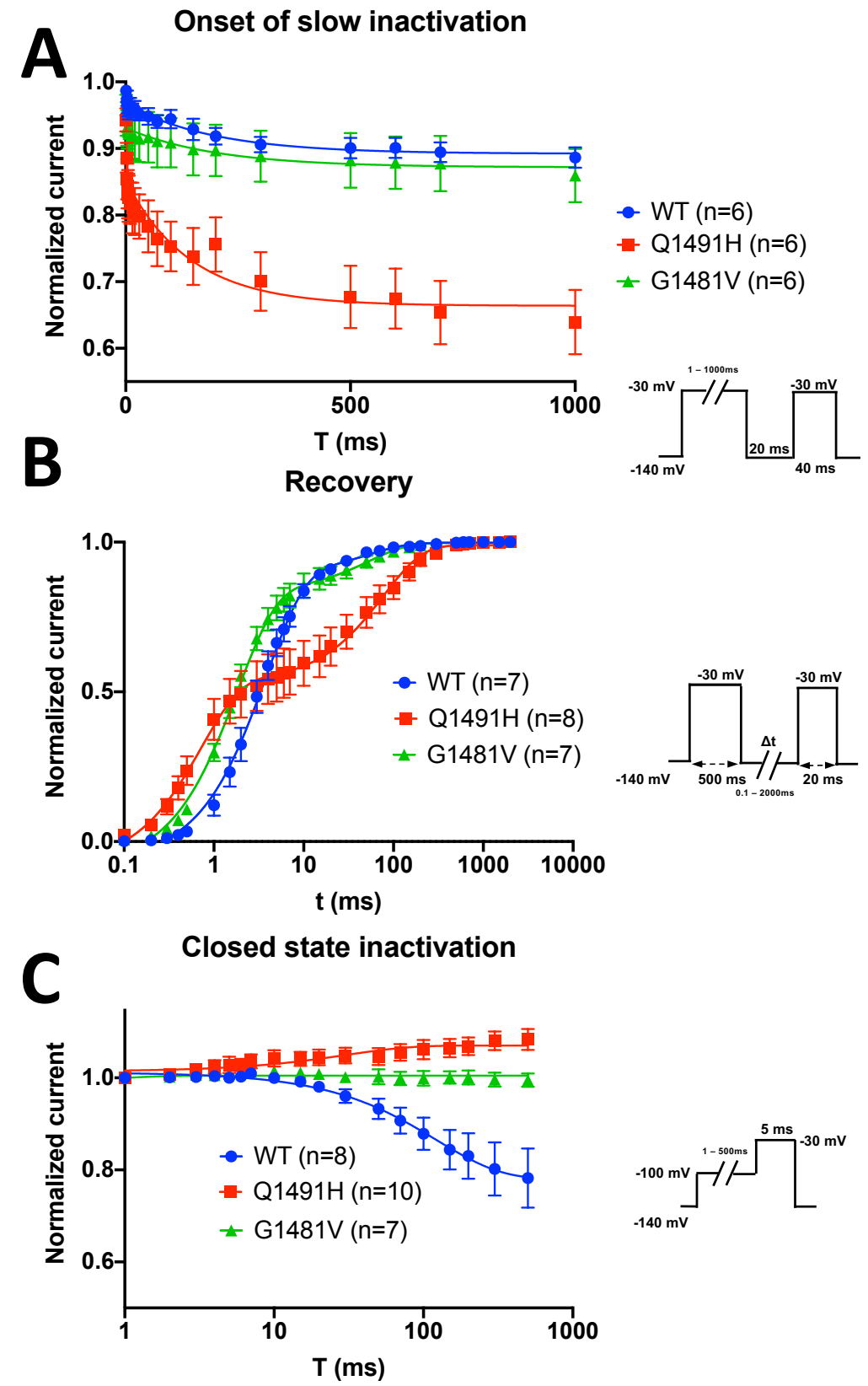


\section{Frequency $2 \mathrm{~Hz}$}
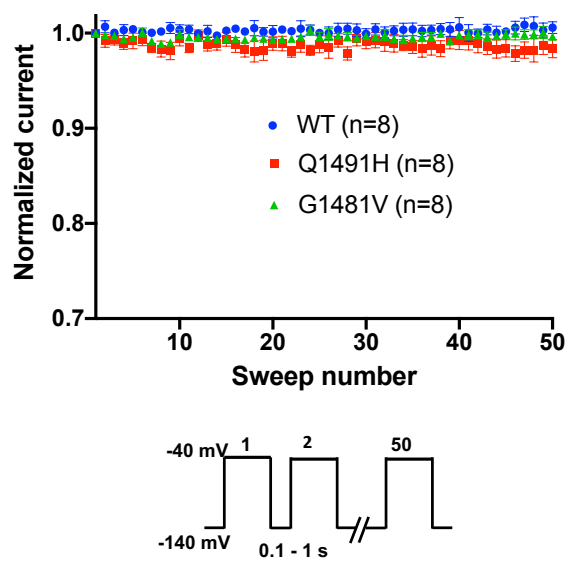

Frequency $5 \mathrm{~Hz}$

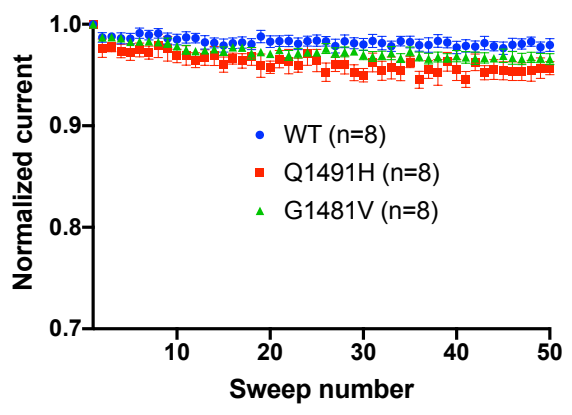

Frequency $10 \mathrm{~Hz}$

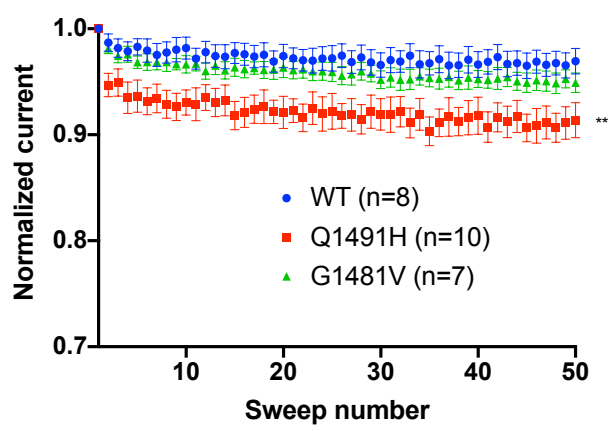



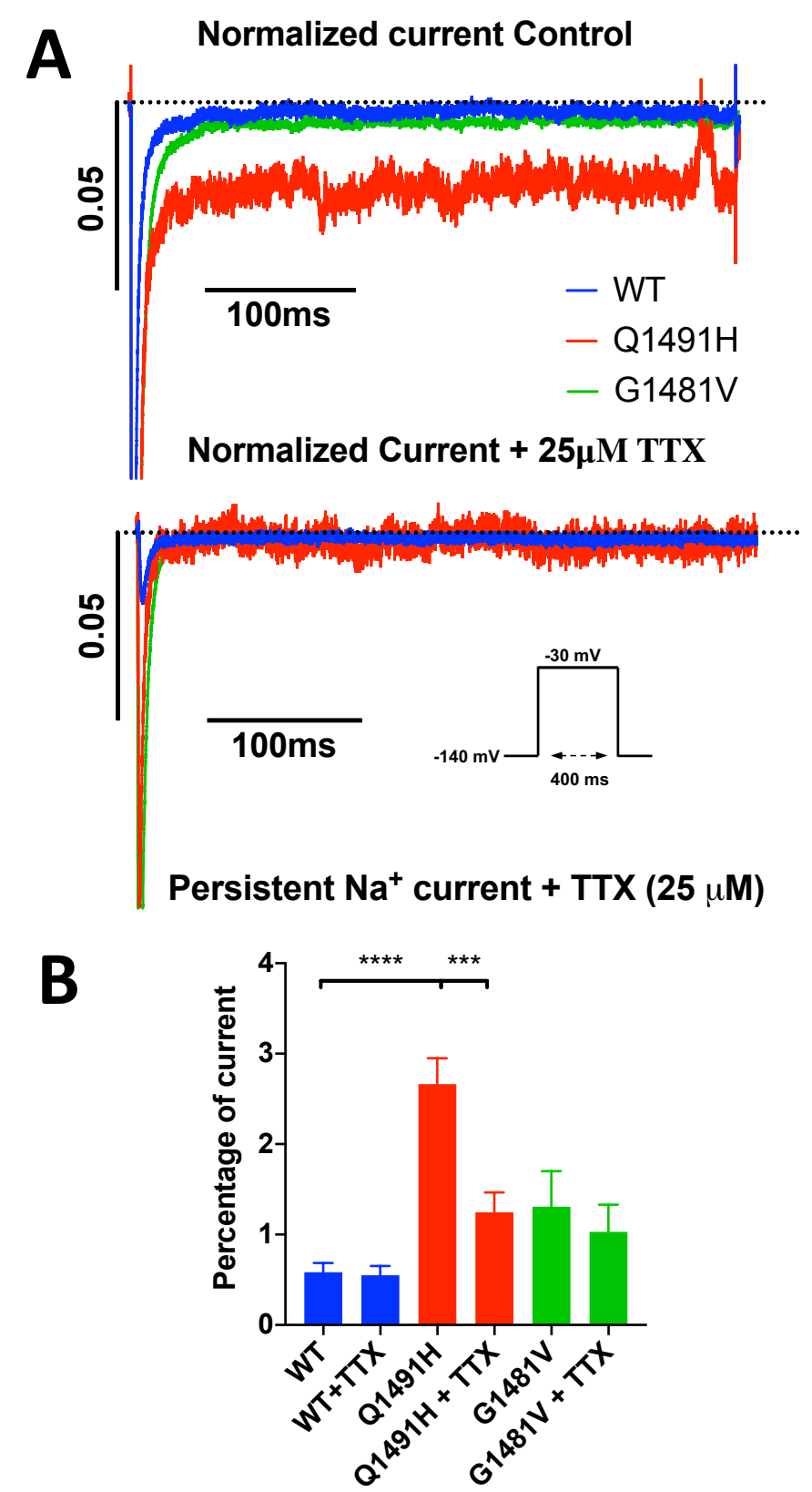
A

Normalized Current with and without $100 \mu \mathrm{M}$ Ranolazine

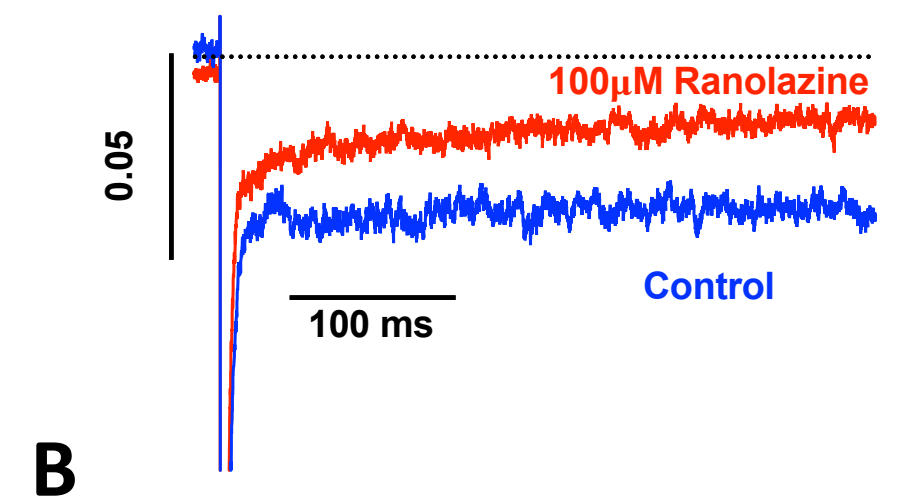

Persistent $\mathrm{Na}+$ current with and without Ranolazine $(100 \mu \mathrm{M})$

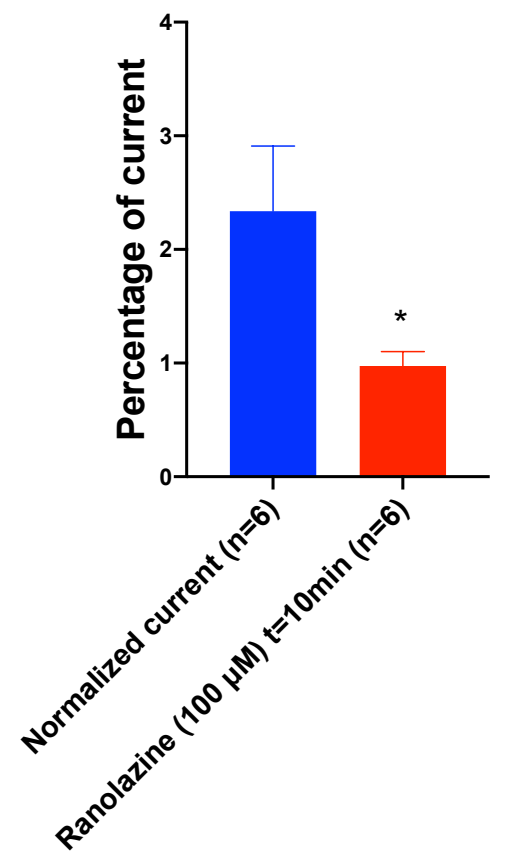




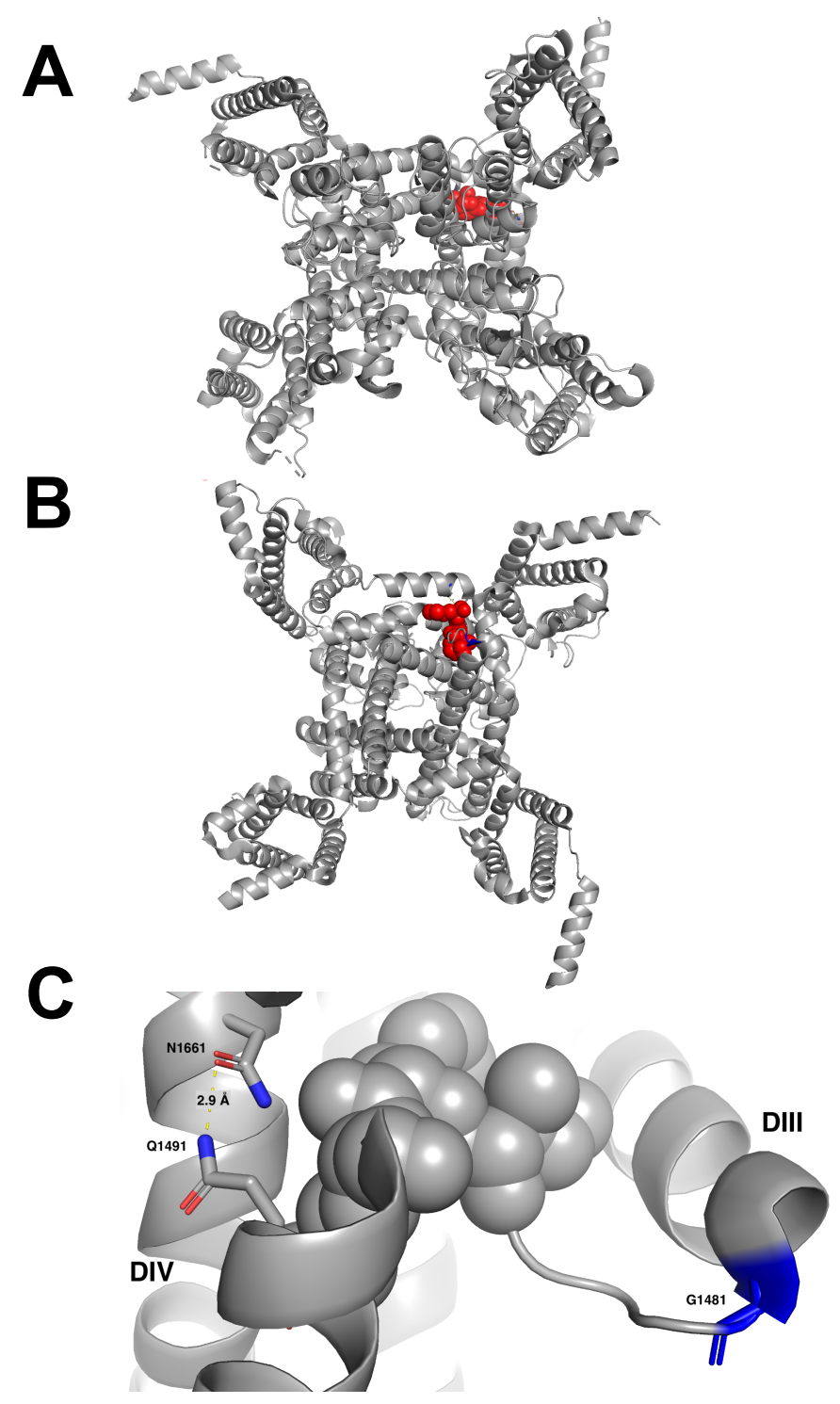




\begin{tabular}{|c|c|c|c|}
\hline \multicolumn{4}{|l|}{ Current Density } \\
\hline $\begin{array}{l}\text { Maximum Peak } \\
(\mathrm{pA} / \mathrm{pF})\end{array}$ & $-235.8 \pm 39.8(n=9)$ & $-49.2 \pm 7.3(n=9)$ & $-391.2 \pm 48.3(n=9)$ \\
\hline \multicolumn{4}{|l|}{$\begin{array}{l}\text { Steady-state } \\
\text { inactivation }\end{array}$} \\
\hline $\mathrm{V} 1 / 2(\mathrm{mV})$ & $-89.6 \pm 1.6(n=9)$ & $-69.4 \pm 1.5 * * * *(n=7)$ & $-82.6 \pm 2.3^{*}(n=7)$ \\
\hline $\mathrm{K}(\mathrm{mV})$ & $-5.1 \pm 0.1(n=9)$ & $-4.5 \pm 0.3(n=7)$ & $-4.6 \pm 0.2(n=7)$ \\
\hline \multicolumn{4}{|l|}{$\begin{array}{l}\text { Steady-state } \\
\text { activation }\end{array}$} \\
\hline $\mathrm{V} 1 / 2(\mathrm{mV})$ & $-41.4 \pm 2.5(n=9)$ & $-32.0 \pm 1.5^{* *}(n=9)$ & $-42.2 \pm 1.2(n=9)$ \\
\hline $\mathrm{K}(\mathrm{mV})$ & $7.642 \pm 1.0(n=9)$ & $14.2 \pm 1.3^{* * *}(n=9)$ & $8.0 \pm 0.6(n=9)$ \\
\hline \multicolumn{4}{|c|}{$\begin{array}{l}\text { Recovery from Slow } \\
\text { inactivation }\end{array}$} \\
\hline$\tau_{\text {fast }}(\mathrm{ms})$ & $4.2 \pm 0.6(n=7)$ & $0.8 \pm 0.1 * * * *(n=8)$ & $1.9 \pm 0.2^{* * *}(n=7)$ \\
\hline$\tau_{\text {slow }}(\mathrm{ms})$ & / & $55.9 \pm 5.0(n=8)$ & $88.2 \pm 12.2(n=8)$ \\
\hline$A_{\text {fast }}$ & $91.0 \pm 1.1 \%(n=5)$ & $56.0 \pm 8.7 \% * *(n=8)$ & $83.2 \pm 4.5 \%(n=7)$ \\
\hline $\mathrm{A}_{\text {slow }}$ & $9.0 \pm 1.1 \%(n=5)$ & $44.0 \pm 8.7 \% * *(n=8)$ & $16.8 \pm 4.5 \%(n=7)$ \\
\hline \multicolumn{4}{|l|}{$\begin{array}{l}\text { Onset of slow } \\
\text { inactivation }\end{array}$} \\
\hline Plateau & $0.9 \pm 0.1(n=6)$ & $0.7 \pm 0.1^{* * *}(n=6)$ & $0.8 \pm 0.1(n=5)$ \\
\hline \multicolumn{4}{|l|}{$\begin{array}{l}\text { Closed State of } \\
\text { inactivation }\end{array}$} \\
\hline Plateau & $0.8 \pm 0.1(n=8)$ & $1.1 \pm 0.1^{* * * *}(n=10)$ & $1.0 \pm 0.1^{* * *}(n=7)$ \\
\hline
\end{tabular}

